\title{
Ocorrência de Molares Supranumerários em Pacientes da Clínica de Radiologia da Universidade Federal do Ceará
}

Occurrence of Supernumerary Molars in Patients from the Radiology Clinic of Federal University of Ceará

George Táccio de Miranda Candeiro ${ }^{1}$, Ana Carolina Santana Praxedes ${ }^{2}$, Amanda Ferreira de Sousa Bringel ${ }^{2}$, Francisco Ramon Ramos Oliveira ${ }^{2}$, Horlley Honny Teófilo Amorim², Lewton de Almeida Fernandes ${ }^{2}$, Ilan Sampaio do Vale ${ }^{3}$

\begin{abstract}
Hyperdontia is a dental anomaly, very common and widely studied, caused by excessive teeth in the oral cavity. This disturb represents an abnormal formation of one or more teeth in both dental arch. The diagnosis is normally based on conventional radiography exams, mainly panoramic radiographies, and in some cases, a computed tomography. In the present study, 1.325 panoramic radiographies were evaluated, all from patients seen by the Radiology Clinic of Federal University of Ceará, residents in Fortaleza, over the period from October 2007 to December 2008. The aim was to evaluate the occurrence of supernumerary molars. Twenty five patients presented supernumerary molars, 13 men and 12 women. However, it was not observed differences statistically significant $(p=0,5264)$. The occurrence in the maxilla was more common compared to the mandible. A considerable occurrence of supernumerary molars in the examined population was reported, reinforcing the importance of a premature diagnosis in this dental anomaly, in order to perform the treatment if necessary.
\end{abstract}

Keywords: Prevalence; Anomalies; Teeth; Diagnostic.

\section{Resumo}

A Hiperdontia é uma anomalia dentária muito comum e largamente estudada, causada por um número excessivo de dentes na cavidade oral. Esse distúrbio representa uma formação anormal de um ou mais dentes em ambos os arcos dentários. O diagnóstico normalmente é baseado em exames radiográficos convencionais, principalmente a radiografia panorâmica, e em alguns casos, a tomografia computadorizada. No presente estudo, 1325 radiografias panorâmicas foram avaliadas, todas de pacientes atendidos na clínica de Radiologia da Universidade Federal do Ceará, residentes em Fortaleza, no período de outubro de 2007 a dezembro de 2008. O objetivo foi avaliar a ocorrência de molares supranumerários. Vinte e cinco pacientes apresentaram molares supranumerários, sendo 13 homens e 12 mulheres. Entretanto, não foi observada nenhuma diferença estatisticamente significante $(p=$ 0,5264). A ocorrência na maxila foi mais comum em comparação à mandíbula. Foi observada uma considerável ocorrência de molares supranumerários na população examinada, reforçando a importância do diagnóstico precoce dessa anomalia dentária, a fim de estabelecer um tratamento, se necessário.

Palavras-chave: Prevalência; Anomalias; Dentes; Diagnóstico.

\author{
${ }^{1}$ Mestrando em Odontologia pela Universidade Federal do Ceará (UFC) \\ ${ }^{2}$ Acadêmicos do curso de Odontologia da Universidade Federal do Ceará (UFC) \\ 3 Professor adjunto da disciplina de Radiologia curso de Odontologia da \\ Universidade Federal do Ceará (UFC)
}

\section{Correspondência: George Táccio de Miranda Candeiro}

Endereço: Rua Elvira Pinho, 288/ casa 02 - CEP 60421-000, Fortaleza - Ceará, Brasil

Fone: (85) 3225-9766

E-mail: georgecandeiro@hotmail.com

Data de Submissão: 27/07/2009

Data de Aceite: 14/10/2009

\section{Introdução}

Uma das anomalias bucais mais comuns são os dentes supranumerários, que são dentes que excedem à quantidade normal, tanto na dentição decídua quanto na permanente (SCHEINER; SAMPSON, 1997; GARVEY; BARRY; BLAKE, 1999).

Os dentes supranumerários são, na maioria dos casos, diagnosticados através de radiografias de rotina, uma vez que são assintomáticos. As radiografias panorâmicas, oclusais e peripicais são úteis na descoberta e na localização dos dentes supranumerários, porém, em algumas situações, exames de imagem com maior capacidade de definição, como a tomografia computadorizada cone-beam, devem ser utilizados a detecção e determinação do correto posicionamento (SAWAMURA; MINOWA NAKAMURA, 2003; BAYRAK; DALCI; SARI., 2005).

Algumas complicações são relacionadas aos dentes supranumerários, como a retenção prolongada do dente permanente, o desenvolvimento de cistos, reabsorções radiculares, o deslocamento dentário e erupções ectópicas (BAYRAK; DALCI; SARI., 2005).

Lee (2006) relatou um caso de um dente supranumerário que teve sua erupção dentro da cavidade nasal, onde acarretava ao paciente dor, deformidade nasal externa, rinorréia e fístula oronasal.

Quanto à classificação Scanlan e Hodges (1997) classificaram dentes supranumerários de acordo com os seguintes critérios: época de erupção, formato e local de erupção. Assim, uma das classificações mais utilizadas faz referência ao local de erupção desses dentes, como incisivos (mesiodens), caninos, prémolares e molares supranumerários.

Os molares supranumerários também são classificados em paramolar, quando o dente encontra-se localizado na região vestibular ou lingual, e distomolar ou $4^{\circ}$ molar, quando o dente estiver localizado na distal do 3ํㅡㄹ (PROFF et al., 2006).

Menardía-Pejuan, Berini-Aytes e Gay-Escoda (2000) relataram 53 molares supranumerários em 36 pacientes, observando que $86,8 \%$ dos dentes estavam na maxila, não apresentando nenhuma relação com a impactação dos terceiros molares.

O diagnóstico desses dentes é realizado, comumente, a partir de radiografias de rotina, uma vez que muitos dentes supranumerários não erupcionam na cavidade bucal, apresentando-se assintomáticos. Assim, radiografias periapicais, 
oclusais e panorâmicas são de grande valia para o diagnóstico, porém não sendo suficientes para o planejamento cirúrgico, pois falham nas informações tridimensionais (BAYRAK; DALCI; SARI., 2005).

Recentemente, a utilização de tomografias computadorizadas tem ajudado no correto posicionamento dos dentes supranumerários. Logo, a partir de reconstruções tridimensionais (3D) e de cortes nos planos sagital, coronal e axial pode-se localizar esses dentes no sentido vestíbulo-lingual facilitando o planejamento. (PREDA et al., 1997; SAWAMURA MINOWA; NAKAMURA., 2003; BAYRAK; DALCI; SARI., 2005; LIU et al., 2007) indicam a utilização rotineira da tomografia computadorizada volumétrica cone-beam (CBCT) como exame por imagem em pacientes com dentes supranumerários.

Assim, o objetivo deste trabalho foi verificar a ocorrência de molares supranumerários em radiografias panorâmicas de pacientes atendidos na clínica de Radiologia do curso de Odontologia da Universidade Federal do Ceará.

\section{Materiais e Métodos}

Para a pesquisa, 580 homens e 745 mulheres, todos residentes em Fortaleza, Ceará, tiveram suas radiografias panorâmicas analisadas, totalizando 1325 pacientes, com o objetivo de avaliar a prevalência de dentes supranumerários. Estes pacientes foram atendidos na clínica de Radiologia do Curso de Odontologia da Universidade Federal do Ceará, no período de outubro de 2007 a dezembro de 2008.

Foi utilizado um aparelho panorâmico Panoura (Yashica, Japão), com miliamperagem e kilovoltagem calibrada de acordo com a massa corpórea de cada paciente, a fim de se obter imagens com máximo detalhe, densidade e contraste médios. Foram excluídos da pesquisa pacientes portadores de alguma síndrome, 0 que representou 15 pacientes.

As radiografias panorâmicas foram analisadas por dois examinadores familiarizados com o aspecto radiográfico de dentes supranumerários com auxilio de um negatoscópio e de uma lupa com 4X de aumento (Kappa=0,93). Em casos de dúvidas, foram realizadas tomadas radiográficas periapicais, para se obter 0 correto diagnóstico.

Os dados foram catalogados em uma tabela que fo utilizada para realizar a contagem e a documentação dos casos encontrados de acordo com o quadrante dentário, a quantidade e a classificação do molar supranumerário (distomolar ou paramolar).

As radiografias que possuíam molares supranumerários foram digitalizadas utilizando uma máquina digital Kodak EasyShare Z650 (Eastman Kodak, Japão), com 6,1 megapixels e 10X de zoom óptico, para posterior análise e confirmação dos resultados.

Os dados foram analisados pelo teste não-paramétrico Qui-quadrado, em relação ao gênero, sendo considerado significante quando $p<0,05$.

\section{Resultados}

Das 1325 radiografias examinadas, $25 \quad(1,9 \%)$ apresentavam molares supranumerários, sendo 13 (52\%) homens e $12(48 \%)$ mulheres, não sendo estatisticamente significante $(p=0,5264)$ em relação ao sexo. Verificou-se que $2,24 \%$ dos 580 homens que participaram da pesquisa e $1,61 \%$ das 745 mulheres possuíam molares supranumerários (tabela 1 ).
Tabela 1. Distribuição dos dentes supranumerários em relação ao gênero na população examinada $(\mathrm{N}=1325)$ e na população afetada pela hiperdontia $(n=25)$.

\begin{tabular}{llll}
\hline & Examinada (\%) & Afetada (\%) & Afetada /Examinada (\%) \\
\hline Gênero & & & \\
Feminino & $745(56,2)$ & $12(48,0)$ & $12 / 745(1,61)$ \\
Masculino & $580(43,8)$ & $13(52,0)$ & $13 / 580(2,24)$ \\
Total & $1325(100,0)$ & $25(100,0)$ & $25 / 1325(1,88)$ \\
\hline
\end{tabular}

Qui-quadrado statístic $=0,4014 ;$ graus de liberdade $=1 ; p=0,5264$

Foram totalizados 35 dentes supranumerários, com uma ocorrência de 33 dentes na maxila $(94,28 \%)$ e 2 na mandíbula $(5,71 \%)$. Houve uma maior freqüência dos distomolares, também conhecidos como $4^{\circ}$ molares, em relação aos paramolares com $62,85 \%$ e $37,14 \%$, respectivamente (gráfico 1 ).

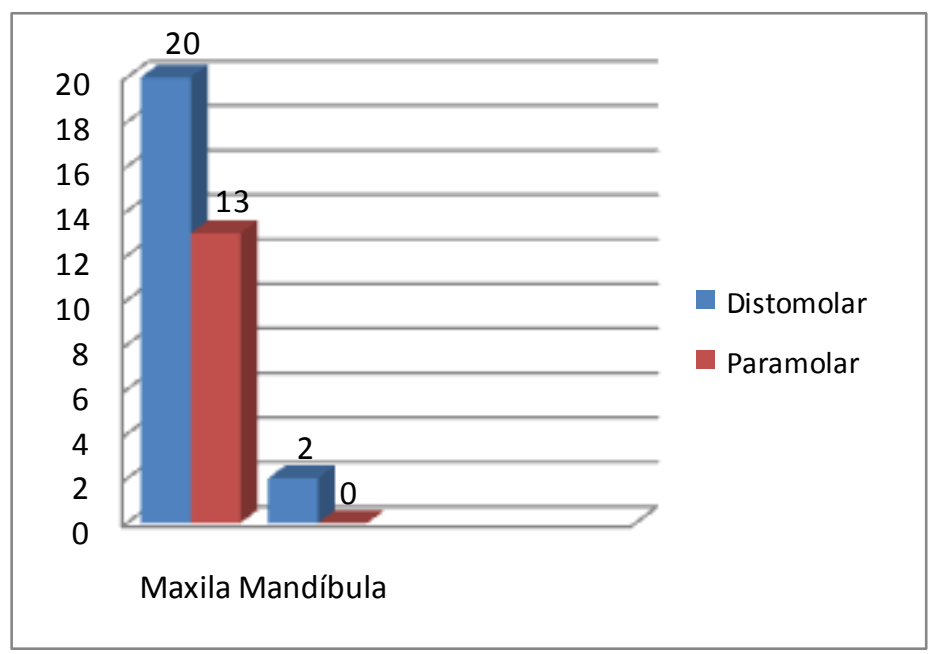

Gráfico 1. Ocorrência de Molares Supranumerários em relação ao arco dentário e a disposição em relação ao 3ํㅡㄹ molar na população afetada pela hiperdontia

Quanto ao quadrante, foram anotados 17 dentes no $1^{\circ}$ quadrante, 16 no $2^{\circ}$ e 1 dente apenas, tanto no $3^{\circ}$ quanto no $4^{\circ}$ quadrantes. Dos pacientes analisados, 17 pacientes possuíam molares supranumerários unilateralmente e oito pacientes bilateralmente. Duas pacientes possuíam dois molares supranumerários, sendo uma na maxila e outro na mandíbula. Houve também uma paciente que apresentava dois molares supranumerários no $2^{\circ}$ quadrante e apenas um no $1^{\circ}$ quadrante.

\section{Discussão}

Os dentes supranumerários são uma das anomalias mais comuns, sendo diagnosticados em exames radiográficos de rotina, uma vez que são normalmente assintomáticos. Sua prevalência varia de acordo com a população estudada, com o gênero e com arcada dentária.

Alguns estudos epidemiológicos mostraram que dentre todos os dentes supranumerários, há uma maior prevelência dos mesiodens (FERNÁNDEZ-MONTENEGRO et al., 2006). Entretanto, outros artigos relatam uma maior freqüência dos molares supranumerários, variando de 37 a $41 \%$ (LEITE SEGUNDO et al., 2006; LECO-BERROCAL; MARTÍN-MORALES; MARTÍNEZGONZÁLEZ, 2007).

Há um predomínio dos dentes supranumerários na maxila, de acordo com Menardía-Pejuan, Berini-Aytes e GayEscoda (2000), Salcido-García et al. (2004), Leite Segundo et al. (2006), Fernández-Montenegro et al. (2006), Leco-Berrocal, MartínMorales e Martínez-González (2007) e Gomes et al. (2008) estando de acordo com os resultados apresentados, 94\% (maxila) e $6 \%$ (mandíbula). 
Assim como os resultados apresentados por SalcidoGarcía et al. (2004) e Fernández-Montenegro et al. (2006), foram encontrados uma maior quantidade de distomolares em relação aos paramolares.

Alguns trabalhos relatam a maior prevalência de dentes supranumerários nos homens, no entanto, não encontramos nenhuma diferença significante entre ambos os sexos, concordando com Grimanis, Kyriakides e Spyropoulos (1991). Salcido-García et al. (2004) relataram uma freqüência de molares supranumerários em $57,1 \%$ das mulheres e $42,9 \%$ dos homens.

Em relação à classificação dos dentes supranumerários, segundo Scanlan e Hodges (1997), entendemos que os molares supranumerários poderiam ser também chamados de quartos molares, e não somente os distomolares. Isso decorre da presença de um dente em excesso em uma região onde deveria haver apenas três dentes, o primeiro, o segundo e o terceiro molares. Sendo que a presença de mais um dente na região de molares originaria um quarto molar. Estes seriam, agora concordando com a classificação de Scanlan e Hodges (1997), subdivididos em disto e paramolares.

Para Azenha et al. (2007), o diagnóstico dessa anomalia é importante para o correto planejamento. De acordo com Hopcraft (1998) e Pawelzik et al. (2002) a radiografia panorâmica é o exame de primeira escolha para o diagnóstico de dentes supranumerários. Farman, Nortje e Joubert (1980), Piatelli e Tete (1992) relatam que em alguns casos pode ser necessário a utilização de outras técnicas radiográficas ou até mesmo o emprego de exames digitalizados, como as tomografias computadorizadas em cortes axiais e coronais para sua exata localização.

\section{Conclusão}

Podemos concluir que os molares supranumerários apresentaram uma considerável ocorrência na população examinada. Dessa forma, é importante o correto diagnóstico por imagens, com a utilização de radiografias panorâmicas e tomografia computadorizada volumétrica, a fim de se realizar o melhor planejamento.

\section{Referências}

AZENHA, M.R. et al. Abordagem cirúrgica de dente supranumerário (Mesiodens) na região palatina: caso clínico. R. Port. Estomatol. Med. Dent. Cir. Maxilofac., Lisboa, v. 48, p. 37-41, 2007.

BAYRAK, S.; DALCI, K.; SARI, S. Case report: evaluation of supernumerary teeth with computerized tomography. Oral Surg. Oral Med. Oral Pathol. Oral Radiol. Endod., St. Louis, v. 100, no. 4, p. e65-e69, Oct. 2005.

FARMAN, A.G.B.; NORTJE, C.J.; JOUBERT, J. Mandibular fourth molars. Ann. Dent., New York, v. 39, no. 2, p. 23-27, Summer 1980.

FERNÁNDEZ-MONTENEGRO, P. et al. Retrospective study of 145 supernumerary teeth. Med. Oral Patol. Oral Cir. Bucal, Valencia, v. 11, no. 4, p. 339-344, July 2006.

GARVEY, M.T.; BARRY, H.J.; BLAKE, M. Supernumerary teeth - an overview of classification, diagnosis and management. J. Can. Dent. Assoc., Ottawa, v. 65, no. 11, p. 612-616, Dec. 1999.

GOMES, C.O. et al. A survey of 460 supernumerary teeth in Brazilian children and adolescents. Int. J. Paediatr. Dent., Oxford, v. 18, no. 2, p. 98-106, Mar. 2008.

GRIMANIS, G.A.; KYRIAKIDES, A.T.; SPYROPOULOS, N.D. A survey on supernumerary molars. Quintessence Int., Berlin, v. 22, no. 12, p. 989-995, Dec. 1991.
HOPCRAFT, M. Multiple supernumerary teeth. Case report. Aust Dent. J., Sydney, v. 43, no.1, p. 17-19, 1998.

LECO-BERROCAL, M.I.; MARTÍN-MORALES, J.F.; MARTÍNEZGONZÁLEZ, J.M. An observational study of the frequency of supernumerary teeth in a population of 2000 patients. Med. Oral Patol. Oral Cir. Bucal, Valencia, v. 12, n. 2, p. 134-138, mar. 2007.

LEE, J. A nasal tooth associated with septal perforation: a rare occurrence. Eur. Arch. Otorhinolaryngol., Heidelberg, v. 263, no. 11, p. 1055-1056, Nov. 2006.

LEITE SEGUNDO, A.V. et al. Estudo epidemiológico de dentes supranumerários diagnosticados pela radiografia panorâmica. $\mathbf{R}$ Cir. Traumatol. Buco-Maxilo-Fac., Camaragibe, v. 6, n. 3, p. 5356, jul./set. 2006.

LIU, D. et al. Three-dimensional evaluations of supernumerary teeth using cone-beam computed tomography for 487 cases. Oral Surg. Oral Med. Oral Pathol. Oral Radiol. Endod., St. Louis, v. 103, no. 3, p. 403-411, Mar. 2007.

MENARDIA-PEJUAN, V.; BERINI-AYTES, L.; GAY-ESCODA, C. Supernumerary molars. A review of 53 cases. Bull. Group. Int. Rech. Sci. Stomatol. Odontol., Valencia, v. 42, no. 2-3, p. 101105, May/Dec. 2000.

PAWELZIK, J. et al. A Comparison of conventional panoramic radiographs with volumetric computed tomography images in the preoperative assessment of impacted mandibular third molars. Int. J. Oral Maxillofac. Surg., Copenhagen, v. 60, no. 9, p. 979-984, Sept. 2002.

PIATELLI, A.; TETE, S. Bilateral maxillary and mandibular fourth molars. Report of a case. Acta Stomatol. Belg., Bruxelles, v. 89, no. 1, p. 57-60, Mar. 1992.

PROFF, P. Problems of supernumerary teeth, hyperdontia or dentes supernumerarii. Ann. Anat., New York, v. 188, no. 2, p. 163-169, Mar. 2006

PREDA, L. Et al. Use of spiral computed tomography for multiplanar dental reconstruction. Dentomaxillofac. Radiol., Houndsmills, v. 26, no. 6, p. 327-331, Nov. 1997.

SALCIDO-GARCÍA, J.F. et al. Frecuencia de dientes supernumerarios en una población mexicana. Med. Oral Patol. Oral Cir. Bucal, Valencia, v. 9, n. 5, p. 403-409, nov./dic. 2004.

SAWAMURA, T.; MINOWA, K.; NAKAMURA, M. Impacted teeth in the maxilla: usefulness of $3 D$ dental-CT for preoperative evaluation. Eur. J. Radiol., Limerick, v. 47, no. 3, p. 221-226, Sept. 2003.

SCANLAN, P.J.; HODGES, S.J. Supernumerary premolar teeth in siblings. Br. J. Orthod., Oxford, v. 24, no. 4, p. 297-300, Nov. 1997.

SCHEINER, M.A.; SAMPSON, W.J. Supernumerary teeth: a review of the literature and four case reports. Aust. Dent. J., Sydney, v. 42, no. 3, p. 160-165, June 1997. 